Thorax (1949), 4, 57.

\title{
CILIARY ACTION
}

BY

\author{
V. E. NEGUS \\ London
}

\section{INTRODUCTION}

To those interested in diseases of the chest, ciliary action should be of absorbing interest, not only through its importance in the interpretation and treatment of inflammatory infections, but also from its intrinsic physiological fascination.

It is surprising that physicians place so much stress on the value of cough and so little on that of ciliary action; the former is really a pathological phenomenon, while the latter is an everyday and normal means of protection. And again, those dealing with respiratory diseases should never lose sight of the dependence on cilia in the physiological processes of the nose and sinuses, with a corresponding danger when the mechanism is upset, the upper air passages then becoming a source of danger instead of a means of protection of the lower air tracts.

\section{Historical}

At a meeting held at the Royal Society of Medicine in 1934, the subject of ciliary action was discussed, and the writer suggested that it.should be looked on as a centenary celebration ; for in 1834 a comprehensive and illuminating article was written by Sir William Sharpey in Todd's " Cyclopoedia of Anatomy and Physiology," published in 1835. Here were described the shape and size of cilia, their mode of action, and their response to disturbing influences. References were made to earlier writings of Purkinje and Valentin, published in 1834.

To define the ciliary pathways, Sharpey inserted charcoal into the antra and trachea of rabbits, and preceded researches repeated in later years.

In 1895 St. Clair Thomson and R. T. Hewlett published an article on the defences of the upper air passages, emphasizing the importance of ciliary action. The subject has been further investigated in more recent years by Gray (1920), Lillie (1906-7), Hill (1928), Lowndes Yates (1924), Proetz (1929-44, Hilding (1932, 1934), Linton (1933), Frenckner and Richtner (1940), Lucas (1931-5), and others. From the work of Proetz, in particular, much of the following information is derived.

\section{Evolution AND Mode of Action}

It is of interest to consider the various purposes to which cilia have been turned in the animal economy. The prototype of protoplasmic protruberances is to be found in pseudopodia which, in such species as Amoeba, serve for both progression and the prehension of food. In these animalculae, as in leucocytes, protoplasmic flow is directed in any needed direction and the broadbased projection acts in the manner of a limb. In ciliated cells, whether in unicellular organisms or in more complicated species, the cilium appears as a permanent projection, with the power of alterfng its shape. It might be thought that flow of protoplasm into a hollow curved tube would explain the movement, but in point of fact there is no evidence to show that a cilium is hollow. Even so, a change in positioning of the protoplasm will lead to a change of shape, converting the resting cilium from a curved to a straight shape, and by the action, if rapidly and forcibly executed, the transition of shape may lead to an even extension, with a bending back beyond a right angle. This is what happens in ciliary action, a whip-like motion leading to an active backward fling, with a passive recoil to the curved resting position. The movement has been compared, also, to that of a fishing-rod, but in either case the analogy is not exact, since in ciliary action there is an active stroke, followed by a passive recoil. The movement of propulsion is in the direction of the active stroke. The rate of beat is said to be 160 to 250 per minute (Frenckner and Richtner, 1940), or even up to 720 per minute (Proetz, 1929-44).

\section{FUNCTIONS}

The purposes subserved by cilia are several and important, even though each cilium is only approximately $7 \mu$ long and $0.3 \mu$ in diameter (Proetz).

Locomotion.-A drop of stagnant pond water reveals Paramoecium briskly darting here and there, propelled by a fringe of actively waving cilia, executing a feathering stroke. Other unicellular organisms have a similar means of movement. 
Deglutition.-The gills of a mussel or an oyster reveal co-ordinated ciliary streams, charged with the function of conveying particles of food to the alimentary system; vorticella shows a similar action, of a beautiful spiral nature. In the frog ciliary streams travel along the walls of the mouth to converge at the mouth of the oesophagus; they continue down the lumen of the latter and are capable of moving particles of a considerable size.

RESPIRATION.-The streams of water conveyed by the cilia of molluscs bring not only nourishment but also oxygen to the gills ; in some fish the respiratory currents are similarly maintained.

Olfaction.-In eels, and some other fish, ciliary action subserves the sense of smell. A stream of water, charged with odours or flavours, is caused to circulate over the olfactory rosette. In man, odours and flavours must go into solution before perception is possible; in this the ciliary streams of mucus play a part in the olfactory area, not only by conveying the stimulus, but also by removing debris such as dust, which might otherwise interfere with olfaction. A similar function is served in those carnivorous or herbivorous animals which rely, far more than man, on the integrity of their sense of smell.

Cleansing.-Cavities with rigid walls, such as the nasal fossae and paranasal sinuses, rely on ciliary action for the removal of debris and bacteria from their lumen ; in collapsible cavities, such as the pharynx, muscular walls are able to expel intruding substances and no cilia are required. It is of interest to note, however, that the upper end of the oesophagus of frogs, and the Fallopian tube in human beings, are lined by ciliated epithelium, even though they are collapsible. There are, of course, limits to the powers of cilia ; it may be observed that when an object too large to be moved is encountered, the ciliary streams pass by on either side, unless obstruction is complete. Some other method of expulsion must then be found, such as sneezing or cough ; but there is a certain degree of filtering of inspired air by the hairs placed in the nasal vestibules, which can not only prevent ingress of over-large objects into the nose but also exclude them from the tracheobronchial tree if inspiration is carried on through the nose only and not through the mouth.

\section{Distribution}

In man cilia line the greater part of the respiratory tract, most of which has an ever-open lumen. But it is unnecessary for there to be a provision of these propulsive agents on every part of the mucosal surface, for, as will shortly be explained, there is no direct action on solid particles but rather a drag on the overlying covering of mucus. It is found that there are no cilia at the extreme anterior part of the nasal fossae-that is, the region in front of the inferior turbinal bodies; nor are there cilia in the air vesicles or atria of the lung. In neither case is this of any disadvantage, because the distal regions referred to can be cleared effectively by a drag on the mucus covering their surfaces (Proetz, 1934).

With the exclusion of the region referred to, all the mucosa lining the nasal fossae and the paranasal sinuses is ciliated ; this motile lining extends into the naso-pharynx and downwards below the level of the Eustachian tubes as far as the upper border of the superior constrictor muscle, which extends well up above the soft palate.

The bronchi are lined everywhere with cilia except for the distal regions mentioned above, the atria, and air vesicles. The trachea is also completely covered, but the larynx has a certain deficiency, since the vocal folds have a squamous epithelium. This means that the only passage for secretions conveyed by ciliary action is through the inter-arytenoid region; any mucus, with entangled debris and bacteria, is tipped over this posterior lip of the larynx and is then removed by swallowing movements.

In the field of this review comes also the middleear tract, which has a ciliated lining, continuous through the Eustachian tabe with that of the naso-pharynx.

\section{Conditions Affecting Normal Ciliary action}

MoIsTURE.-Cilia cannot work alone; they do not propel debris or bacteria directly, and cease to work if the mucosal surface becomes dry. It is essential that there should be a covering of mucus, or "blanket" as Proetz (1941) has described it. The whole mechanism may be compared to a conveyor belt or to a moving staircase or an escalator, in which the platform on which passengers stand or conveyed packages rest corresponds to the blanket of mucus ; the propulsive agent of underlying pinions is represented by the cilia, which thus propel and remove deleterious particles.

The blanket of mucus must be of the right viscosity, sufficiently tenacious to cohere, but not so tenacious as to clog the cilia; this faculty of cohesion enables cilia to work in relays, because a layer of secretion can be pulled from a distance. The mucous sheet is composed of an outer stratum of mucus resting on the tips of the cilia and an inner fluid layer of low viscosity, forming a suit- 
able medium for the vibrating cilia (Lucas and Douglas, 1934).

In sinuses it is the cilia of the lining membrane, with their propulsive power, which carry secretions as far as the ostium, but it is apparently the dragging effect of the epithelium in the nasal fossae which executes the next stage of extracting secretions and conveying them into the naso-pharynx. Similarly, the cilia of the terminal bronchioles can cleanse the air sacs of the lung.

Not only must the viscosity of the mucus conform to certain criteria, but there must also be a correct quantity, neither so great as to fill a bronchiole or sinus, nor so deficient as to lead to stagnation.

The sensitivity of response to these alterations in quantity may be observed in experimental animals ; general anaesthesia may so diminish the total quantity as to slow up or stop the streams, and it may be found necessary to give pilocarpine to increase the supply, or, alternatively to make use of decerebrate animals.

The viscosity of secretions may be affected by local applications; glycerine or oil may make it too thick, or potassium iodide may reduce it to too thin a consistency. In the former case there is stagnation and lack of movement, and, in the latter, lack of concerted action.

Not only does this concerted synergism between cilia and mucus allow of cleansing of non-ciliated areas, but it also enables rest to be afforded to certain groups, while other cilia maintain streaming of the superjacent blanket (Proetz).

The viscosity and quantity of secretion must be adjusted, if the mechanism of protection is to be maintained, in accordance with variations in the atmosphere; at high altitude or in steam-heated rooms, the air is dry and greater calls are made on the secretory mechanism.

TEMPERATURE.-Unless extreme, variations of temperature have little effect on ciliary action, unless of course the overlying secretion becomes frozen or removed by desiccation (Proetz, 1934). Cilia work efficiently in the mouth of the frog and the gills of the oyster, as well as in the trachea or bronchi of the rabbit. And in man there is no lapse in the protective mechanism, since the cilia of the mucosa covering a mass of adenoids, or that over nasal polypi, will continue to function after removal from the body. Even after death of the body as a whole, there is a continuation of ciliary streaming at a time when the body temperature has dropped to that of the atmosphere.

It may be concluded that the deleterious effects of moderate changes of temperature are due to the increase or reduction of mucus, and not to any direct influence on the ciliated epithelium.

SLEeP.-If cilia continue to work after death of the remainder of the organism, it is no cause for surprise that they maintain their efficiency during sleep; there is a team action of work by relays, but no cessation of protection.

ReAction of Medium.-There are here two aspects to be considered; one is the medium in which the cilia are immersed, the other is the nature of the tissue fluids.

(a) Secretions.-To consider the former first, as being of the lesser importance, it is to be noted that considerable divergencies of opinion exist as to the normal reaction of mucus, a word used somewhat loosely. Mucus, as applied to the secretions of the nose and bronchi, is a mixture made up partly of the secretion derived from goblet cells and racemose mucous glands, together with transudate from the capillaries, escaping from the epithelial cells or through intercellular spaces together with tears. The proportion of the two components is capable of wide variation, with a consequent alteration in viscosity and reaction. The powers of secretion and transudation are so versatile as to be capable of adjusting the blanket of mucus to correspond with alterations of atmospheric conditions, and, in fact, the physiological variations very easily merge into pathological abnormalities; this question will be considered later. What is in question at present is the reaction of the mixed secretion.

Estimations of reaction may be carried out by various methods-colorimetric, electrometric, or otherwise-and the means used for testing may lead to mistakes. If nasal secretion be removed from the body and tested, readings of the $p \mathrm{H}$ are round about 7.4 , but if estimations are made in situ, as described by Fabricant (1941), a lower figure of about 7.0 is found (see also Tweedie, 1934). The matter is not of vital importance, and the explanation of the small discrepancies is not difficult to discover; for normal mucus contains some easily dissoluble carbon dioxide, which is readily given off when the secretion is exposed to the outside air. If any interval is allowed to elapse, therefore, long enough for loss of carbon dioxide, the reaction will be less acid than when the estimation is made in situ. There is known to be an active excretion of carbon dioxide from the nasal mucosa ; it is, in fact, a mechanism of nasal respiration (Hellmann, 1926). The escape of carbon dioxide is said to be seven times more active from the nasal than from the alveolar epithelium, and 
the influence on the reaction of the nasal secretion is considerable.

Inquiry must be made as to whether these discrepancies of estimation have any marked physiological importance; unless extreme, this does not seem to be the case. Cilia work actively when in a neutral medium, and possibly rather more briskly still when the reaction is raised, even to $p \mathrm{H} 8.5$; but, on the other hand, full functional activity has been observed by Proetz in ciliated epithelium removed from an infected maxillary sinus, in which glycophilic bacteria, producing lactic acid, had lowered the reaction to a $p \mathrm{H}$ well below 7.0.

Although the reaction of the free secretions may have an influence which is slight if moderate variations occur, yet it is rather on the reactions of the inter- and intra-cellular fluids that efficiency of action depends. Mittermaier (1930) found that the $p \mathbf{H}$ of the tissues does not of necessity correspond with that of the secretions.

(b) Tissue fluids.-Conditions here are somewhat different from those in the lumen of a sinus or bronchus. While little effect is brought to bear on the cilia themselves by changes of medium, yet much influence is felt when these changes affect the tissue fluids. For the efficient nourishment of columnar cells bearing cilia, two essentials must be satisfied; these are the correct proportion of elements and also the correct reactions of the fluids.

The supply of necessary ions has been studied by Gray, Lillie, and others ; it is found that there must be present, in correct proportions, sodium, calcium, potassium, manganese, phosphorus, and ammonium. These elements can be combined in a solution such as that of Tyrode or Ringer ; ciliary action under experimental conditions will continue almost indefinitely in these media, but if there be any deficiency the activity gradually diminishes and finally ceases.

Normal saline is often referred to as a physiological solution, but it is quite inadequate to maintain the activity of excised ciliated epithelium, which, if placed in such a medium, rapidly loses its activity and soon ceases action.

On the other hand, the right admixture of ions must be related to a correctness of reaction; this is determined mainly by the concentration of hydrogen ions. However little may be the effect of alterations of $p H$ on the cilia themselves, yet a different result is found when similar changes affect the tissue fluids. For if excised ciliated epithelium be kept in an artificial medium it will be found that a rise to the alkaline side, with a $p \mathrm{H}$ of over 8.0, tends to cause swelling of the cells due to increased permeability of the cell walls; and in extremes the cells swell up and cease to function. On the other hand, a lowering of $p \mathbf{H}$ to below 7.0 leads to shrinkage of the cells and diminution of activity.

In pathological states, changes in reaction of the exudate are not necessarily correlated with alteration in the $p \mathbf{H}$ of the intercellular tissue fluids, and consequently no loss of activity may be observed. It is possible for the secretions in the epithelial surface to penetrate and to affect the cells ; penetration of vegetable acids such as lactic and formic have been shown by Gray to produce a depressing effect, while mineral acids have little action, owing to lack of passage into the cells.

But the reaction of the tissue fluids is subject to fluctuation; with an increase of the alkali reserve or with increased excretion of carbon dioxide from the nasal epithelium, there may be a corresponding rise towards the alkaline side, with consequent changes in ciliary activity. The question will be returned to later.

Osmotic Pressure.-The influence of changes in this respect has already been covered, more or less, in connexion with the supply of ions and the concentration of hydrogen ions. Osmotic pressure must be so balanced as not to lead to oedema or to dehydration, both of which impair the activity of epithelial cells. In experimental investigations it is essential that the medium used should be isotonic, if functional activity is to be maintained.

EFFECT OF Gravity.-Experiments show that change of position has no appreciable effect on the rate of ciliary streams. The question is one of importance, because it might be thought that the drainage of the nose and sinuses would be more efficiently carried out than that of the trachea and bronchi. From personal observation, this is not the case, since gravity appears to have no effect on ciliary action. Thus in one experiment the time taken to travel $1 \mathrm{~cm}$. was 33 seconds in an upward direction, 26 seconds on the level, and 34 seconds downwards.

RATE of Progress.-In the nose the estimated rate is $1 \mathrm{~cm}$. in half a minute, or $2 \mathrm{~cm}$. a minute (Proetz). In the bronchi progress is slower, at $0.25 \mathrm{~cm}$. per minute (Proetz). Florey and others (1932) found the rate to be $3.5 \mathrm{~cm}$. in one minute in the cat's trachea.

Direction of Streams.-The pathways of ciliary streams are fixed and unchangeable (Lucas, 1933). In the sinuses they are all directed towards the ostium and therefore the course may be lengthy, since some of the streams 
must pass outwards across the floor, upwards on the outer wall, and inwards across the roof before reaching their point of exit.

In the trachea there is a serpentine movement at the bifurcation, according to the observations of Barclay and others (1937). This is to be expected, since the streams from the two bronchi meet and coalesce. When the larynx is reached, the track is towards the posterior commissure, since there are no cilia on the vocal folds; the latter regions, being covered with squamous epithelium, cannot propagate ciliary streams.

It is interesting to note the reported observation that cilia always drive towards the point from which they originated. If the lining of the maxillary sinus be removed, with regeneration of a new one from ingrowth and spreading in from the ostium, the new streams will always be directed towards the point of regeneration - that is, the ostium.

Propagation of Impulse.-An isolated epithelial cell has four or five ciliary projections, which continue to beat if in a suitable medium. No nervous stimulation is at work, and apparently none is required, the rate of beat being an inherent attribute of the cell (Lucas, 1931). Propagation through the cytoplasm from cell to cell is sufficient to maintain an orderly sequence of action, as may be observed in a fragment of the gill of an oyster, in which a wave motion flows along the line of cilia. Nerve impulses may activate ciliary movement, but they do not inhibit it ; impulses pass by the parasympathetic portion of the autonomic system (Lucas, 1935).

Powers of Resistance.-The recuperative powers are considerable under changes of temperature or under experiments with various drugs. Unless killed by excessive heat or by powerful agents, such as cocaine of 10 per cent strength, a restoration of favourable conditions is followed by renewal of ciliary action. More will be said later on this point.

REGENERATION.-It has been noted by Knowlton and McGregor (1928), Gorham and Bacher (1930), and Hilding (1933) that ciliary epithelium may regenerate, both experimentally in animals and after operation in man, when the lining of a sinus has been removed. Regrowth must not, however, be relied upon, particularly if scar tissue forms in the submucous layers (Hilding, 1932). Operations for removal of adenoids are naturally associated with denudation of a considerable area of the naso-pharyngeal vault, but if performed without undue injury to the underlying aponeurosis, replacement may be expected. But if rough curettage has been performed scar tissue may be produced, with consequent prevention of ciliary regeneration; in such a case debris and bacteria, carried back from the nose and sinuses, may stagnate in the naso-pharynx and lead to droplet infection of the tracheo-bronchial tree.

In cases of acute rhinitis, shedding of the surface mucosa may occur, followed, according to reports, by regeneration in a few hours under favourable conditions. Tissue culture of ciliated epithelium has demonstrated the outgrowth of cells with motile projections (Lucas, 1933 ; Proetz and Pfingsten, 1939).

ForCE EXERTED.-The strength of ciliary action is very considerable, as may be observed by placing . some pieces of thick paper in the roof of the mouth of a frog. In experiments with excised pieces of epithelium, viewed on edge, the propulsion of blood corpuscles, used as indicators, is very striking.

Hilding (1944) has measured the changes of pressure produced during the expulsion of a plug of mucus from the trachea of a hen, and has found it reduced to $40 \mathrm{~mm}$. of water. He concludes that such an action may play a part in deflation of the lungs and may be instrumental to a certain degree in producing the changes known to occur after plugging of a bronchus in man. Massive collapse may follow, even if not occurring before the plugging, especially after operations in the upper abdomen.

Experimental observations show that cilia may continue to wave without creating a marked propulsive effect, owing to a decrease of amplitude of the stroke.

\section{Conditions Producing Disordered Action}

DRYING.-At the beginning of a cold in the nose, probably through the action of the causative virus, areas of mucosa, usually far back in the fossa or on the upper surface of the soft palate, become dry. When this occurs ciliary action ceases at the margin of the dry patch ; any bacteria carried back are left to stagnate and to multiply. The mechanical action of the ciliary streams is interrupted, and no longer are pathogenic organisms conveyed to the naso-pharynx, to be removed thence by peristaltic contraction of the superior constrictor, followed by a swallowing movement travelling down the pharynx to the oesophagus. Furthermore, the absence of mucus removes the protective action of lysozymes, described by Fleming (1928); the inhibitory or 
bactericidal action of the secretions from the nose and sinuses-and the tears from the eyes-is no longer available to check the growth of the secondarily invading organisms. Drying is also caused by residence in overheated and undermoistened rooms, when air-conditioning is inefficient; it appears also in the dry atmosphere of Western Australia and in the high altitudes of the Himalayas. Atropine may do harm by reducing the quantity of secretions in the nose and tracheobronchial tree if given in an amount greater than required to counteract the stimulating effect of such an anaesthetic as ether. Atrophy of the nasal mucous membrane is accompanied by reduction in the amount of secretion and transudate, with consequent infection. Desiccating agents such as - chemical corrosives and caustics, the galvanocautery, or diathermy, dry the surface but may also destroy the cilia and produce scar tissue which prevents regeneration.

SWelling of Epithelium.--It is found experimentally that an excessive rise of the $p \mathrm{H}$ is accompanied by swelling of the epithelial cells, with final cessation of action. Excess of one ion and the absence of others may produce a similar effect; sodium tends to lead to swelling, and potassium and calcium to shrinkage, all with disturbance of ciliary movement.

Mechanical Obstruction.-Blockage of the nasal fossae or of the ostium of a sinus by inflammatory or allergic swelling or other cause interrupts ciliary action; similar changes may occur in an inflamed bronchus or in one plugged by an inhaled.foreign body or by a plug of viscid mucus.

ITERRUPTION OF STREAM.-The presence of a small foreign body in a bronchus, even of insufficient size to cause plugging, may encourage the appearance of granulations; these have no cilia on their surface, and the ciliary stream is thereby interrupted and secretions pile up on the distal side. This may occur in cases of bronchial or lung abscess.

A drop of chloroform condensing in the trachea may inhibit a band of cilia, thus interrupting the streams completely and possibly permanently.

As mentioned above, destruction of part of the ciliary chain by coagulation, effected by the galvano-cautery or by surgical diathermy, is another means of upsetting the mechanism. Similarly, removal of the epithelium at operation may have the same effect; this may occur as the result of removal of adenoids. When the lymphoid mass is shaved off, the covering epithelium must of necessity be removed; regeneration may be complete and effective unless there has been damage to the underlying aponeurosis or muscles. In the latter case the formation of scar tissue is likely to prevent the regrowth of cilia. Operations on the sinuses should be designed to interfere as little as possible with ciliary action; the making of high and narrow openings into the maxillary or sphenoid sinuses is therefore preferable to the creation of long windows (Proetz).

EFFECT OF Drugs.-Apart from the actual destruction by such an agent as liquid chloroform, there are frequent occasions on which the incorrect use of medicaments has a harmful effect (Negus, 1934).

Cocaine in concentrated solution inhibits cilia ; 2.5 per cent solution is almost harmless if applied for short periods, but 10 per cent causes cessation of action, while stronger concentrations are said to be destructive. This observation is, however, open to doubt, since patients to whose nasal mucosa 25 per cent paste, or cocaine mud, has been applied do not seem to suffer eventual bad effects. Drying agents like atropine, in excess, are harmful, in that they remove the blanket of mucus without which cilia cannot function; in operations on the larynx, in particular, this drug should not be abused, for fear of subsequent infection of the inefficiently protected tracheo-bronchial tree. Ephedrine in half or three-quarter per cent strength has no direct ill effect, unless it dries the sccretion overmuch. Thymol is harmful, and so are most antiseptics except those of the silver group, such as argyrol and protargol in 2.5 or 5 per cent strength. Not only may there be a deleterious effect by chemical action but also one of a direct physical nature. Thus glycerine in strength makes the blanket of mucus too viscid, while oils, although not mixing with the watery medium, have an entangling and impeding effect.

What is spoken of as physiological saline solution is sometimes found in practice to be anything but beneficial if nasal douching is employed, since there is not only washing away of the blanket of mucus, if the liquid is used too freely, but also an inhibitory effect, probably due in the main to desia cation; the addition of borax or phenol to collunaria does nothing to obviate these objections ; but, if used judiciously, isotonic solutions made up with sodium chloride are useful and harmless if used in sprays.

Changes in Reaction of Medium.-The necessity for the supply of a variety of ions in correct proportion has already been referred to, but in addition there must be an accurate concentration of hydrogen ions ; this means, of course, a reac- 
tion of the right order. This observation refers to the epithelial cells and tissue fluids rather than to the superficial secretions. Examinations of the $p H$ of the tissue fluids and the secretions have been made by many investigators and by various methods, with varying results, as already described. In point of fact, the reaction of the medium on the surface of the epithelium has no great effect on the activity of cilia. Experiments show that cilia work actively in a medium just below the neutral point, and even at as low a level as 6.5. On the other hand, a rise of $p \mathbf{H}$ to 8.0 or 8.5 is still associated with active ciliary action; but such a rise occurs in the early mucoid stages of acute rhinitis, with a lowering of resistance to bacteria (Hilding, 1934). In the later stages of suppuration in the nose or sinuses the reaction drops to below the neutral point, even to $p \mathrm{H} 5.86$, according to Buhrmester (1933). Ciliary action may continue in an infected sinus (Proetz, 1933).

In life extreme variations do not occur, but small changes due to alterations in the alkaline reserve, or in the excretion of carbon dioxide, may affect the action of cilia. Luscher (1930) has shown that the alkaline reserve is lowered where there is nasal obstruction; no doubt the diminished excretion of carbon dioxide accounts for this change. In allergic states the $p \mathrm{H}$ rises, but infections are not usual; it may be concluded from this that ciliary action is not deleteriously affected (Kreewinsch, 1932). The production of lactic acid by glycophilic bacteria has been referred to, with some possible effect on the epithelial cells. Vegetable acids such as lactic acid and formic acid penetrate cell walls more readily than mineral acids such as hydrochloric; their effect on cell activity may therefore be felt.

\section{Relationship Between Upper and Lower Air Passages}

Many particles of dust and many bacteria are prevented by the nose from entering the lower air passages, provided, of course, that nasal respiration is maintained. Breathing through the mouth owing to nasal obstruction, or keeping the mouth open when talking, naturally allows unfiltered air to enter the tracheo-bronchial tree. But if inspiration is effected through the nose, there is a considerable degree of filtering. This depends first on the presence of vibrissae in the nasal vestibule, secondly on the entangling influence of slightly viscid nasal mucus, and thirdly, as Proetz has described, on surface attraction of particles by static charges. The secretions, with their contained debris, are dumped in the naso-pharynx, to be removed by the swallowing mechanism. But even so, many bacteria must be carried down with the inspired air during nasal respiration, and more during mouth-breathing. The ejection of these from the bronchi and trachea is carried out by the local ciliary streams. And in cases of laryngectomy, when the trachea opens directly on the front of the neck, the lower passages are able to remain in a state of health by virtue of their own defences.

The ciliary mechanism of the nose is of assistance in protecting the lungs, but alone it is not sufficient and must be supplemented by the defences of the tracheo-bronchial tree. Furthermore, it may be added that when the nasal or naso-pharyngeal mechanism is deranged, the nose may become a danger instead of a protection to the lungs.

\section{CONCLUSIONS}

To preserve ciliary action, and to assist its highly important function of resisting infection, it is necessary to be acquainted with all the factors outlined above, and to take such measures in the prevention and treatment of disease as appear of assistance to physiological processes. Thus the blanket of mucus must not be washed away by nasal douches, nor must it be made too viscid by concentrated glycerine, nor too liquid by excessive use of potassium iodide. The secretions must not be dried by hypertonic solutions, by excessive use of amphetamine or atropine, or by residence in an inefficiently conditioned building. Secretions must not be allowed to accumulate in a sinus or bronchus; they must be removed, if excessive, by washing out or aspiration. No drugs must be employed, except for brief periods, which have a toxic effect on the mucosa ; cocaine, thymol, and most powerful antiseptics are in this category.

Obstruction to ciliary streams must be cleared, whether a plug of coagulated muco-pus in the ostium of the maxillary sinus, a foreign body, or a mass of new growth in a bronchus; similarly, a septal deviation may need correction.

If an operation is performed in an area covered by ciliated epithelium, every care must be taken to avoid damage of a permanent nature. Thus, adenoids must be removed in such a way as to avoid scar-tissue formation and to allow of regeneration; in a sinus the opening must not be overwide, nor should the mucosa be removed if capable of recovering and of once again serving its physiological functions, nor must cauterization be applied to wide areas for fear of scarring.

The reaction of the secretions must be regulated where possible to facilitate free action; a $p \mathrm{H}$ just above neutral appears best, and for this 
reason spray solutions are made slightly alkaline. The alkali reserve of the body may require correction on occasion.

If all these points are kept in mind, and if ciliary action maintains its efficiency, then there will be no penetration of the mucosa by bacteria and no strain will be thrown on the leucocytic second line of defence.

\section{REFERENCES}

Barclay, A.E.,Franklin, K. J., and Macbeth, R.G. (1937). J. Physiol., 90, 347.

Buhrmester, Catherine C. (1933). Ann. Otol. etc., St Louis, 42, 1041.

Fabricant, N. D. (1941). Arch. Otolaryng., Chicago, 34, 150.

Fleming, A. (1928). J. Laryng., 43, 385.

Florey, H., Carleton, H. M., and Wells, A. Q. (1932). Brit. J. exp. Path., 13, 269.

Frenckner, P., and Richtner, N. G. (1940). Acta otolaryng., Stockh., 28, 215.

Gorham, C. B., and Bacher, J. A. (1930). Arch. Otolaryng., Chicago., 11, 763.

Gray, J. (1920). Quart. J. micr. Sci., 64, 345.

Gray, J. (1921-2). Proc. R. Soc. B, 93, 104; 93, 22.

Gray, J. (1928). "Ciliary Movement." London: Macmillan and Co.

Hellmann, K. (1926). Z Z. Laryngol. Rhinol., 15, 1.

Hilding, A. (1932). Arch. Otolaryng., Chicago, 15, 92.

Hilding, A. (1933). Arch. Otolaryng., Chicago, 17, 760.

Hilding, A. (1934). Ann. Otol, etc., St Louis, 43, 47.

Hilding, A. C. (1944). Anesthesiology, 5, 225.

Hill, L. (1928). Lancet, 2, 802.

Knowlton, C. D., and McGregor, G. W. (1928). Arch. Otolaryng., Chicago, 8, 647.

Kreewinsch, P.(1932). Acta oto-laryng., Stockh.,17,48.
Lillie, R. S. (1906-7). Amer. J. Physiol., 17, 345;

Linton, C. S. (1933). Ann. Otol., etc., St Louis, $42,64$.

Lucas, A. M. (1931a). J. Morph. Physiol., 51, 147.

Lucas, A. M. (1931b). Trans, Amer. laryng. rhin. otol. Soc., 37, 172 .

Lucas, A. M. (1933). Proc. Soc. exp. Biol., N.Y., 30, 501.

Lucas, A. M. (1933). Arch. Otolaryng., Chicago, 18, 516.

Lucas, A. M. (1935). Amer. J. Physiol., 112, 468.

Lucas, A. M., and Douglas, L. C. $(1933-4)$. Proc. Soc. exp. Biol., N.Y., 31, 320.

Lucas, A. M., and Douglas, L. C. (1934). Arch. Otolaryng., Chicago, 20, 518.

Lüscher, E. (1930). Acta oto-laryng., Stockh., 14, 90.

McGregor, G. (1931). Arch. Otolaryng., Chicago, 14, 309.

Mittermaier, R. (1930a). Arch. Ohren-Nasen- u. Kehlkopfheilk., 126, 390.

Mittermaier, R. (1930b). Z. Laryng. Rhin. Otol., 19, 390.

Negus, V. E. (1934).' J. Laryng., 49, 571.

Proetz, A. W. (1929). Ann. Otol., etc., St Louis, 38. 963.

Proetz, A. W. (1932a). Ann. Otol., etc., St Louis, 41, 125.

Proetz, A. W. (1932b). Ann. Otol., etc., St Louis, 41, 1117.

Proetz, A. W. (1933). Ann. Otol., etc., St Louis, 42, 778.

Proetz, A. W. (1934). J. Laryng., 49, 557.

Proetz, A. W. (1941). "Applied Physiology of the Nose." Annals Publishing Co. St. Louis.

Proetz, A. W. (1944). Arch. Otolaryng., Chicago, 39, 514.

Proetz, A. W., and Pfingsten, M. (1939). Arch. Otolaryng., Chicago, 29, 252.

Purkinje, J. E., and Valentin, G. (1834). Arch. Anat. Physiol., Lpz., p. 391.

Sharpey, W. (1830). Edinb. med. surg. J., 34, 113.

Sharpey, W. (1835a). Edinb. new philo. J., 19, 114.

Sharpey, W. (1835b). Article on cilia in Todd, R. B., "Cyclopaedia of Anatomy and Physio!ogy." London.

Thomson, St. Clair, and Hewlett, R. T. (1895). Med.chir. Trans., 78, 239.

Tweedie, A. R. (1934). J. Laryng., 49, 586.

Yates, A. Lowndes (1924). J. Laryng., 39, 554. 